\title{
Application of dual-source CT perfusion imaging and MRI for the diagnosis of primary liver cancer
}

\author{
DONGWEN ZHANG and AIXIA XU \\ Imaging Center, Affiliated Hospital of Weifang Medical University, Weifang, Shandong 261031, P.R. China
}

Received January 25, 2017; Accepted April 25, 2017

DOI: 10.3892/ol.2017.6170

\begin{abstract}
The objective of the present study was to evaluate the application of dual-source CT perfusion imaging and MRI for the diagnosis of primary liver cancer. Sixty-three patients with primary liver cancer were selected between February 2015 and May 2016. All patients underwent examinations by dual-source CT perfusion imaging and MRI. The perfusion parameters of the focus center and normal liver parenchyma by CT examination and the hemodynamic parameters of the focus center and normal liver parenchyma by MRI examination were analyzed. The accuracy rates of the three detection methods (CT perfusion imaging, MRI, and combined examination) were analyzed and compared by ROC curves. Dual-source CT perfusion imaging revealed that blood flow and blood volume of the focus center were significantly higher than those of normal liver parenchyma $(\mathrm{P}<0.05)$. MRI examination showed that hepatic arterial perfusion and hepatic perfusion index of the focus center were significantly higher than those of normal liver parenchyma; portal venous perfusion of the focus center was significantly lower than that of normal liver parenchyma $(\mathrm{P}<0.05)$; the difference in total liver perfusion between the focus center and normal liver parenchyma was not significant $(\mathrm{P}>0.05)$; the accuracy rates of $\mathrm{CT}$ perfusion imaging, MRI, and combined examination were $76.19,85.71$, and $95.24 \%$ respectively; the area under the curve of CT perfusion imaging was $0.753(\mathrm{P}<0.05)$, the sensitivity was $79.2 \%$ and the specificity was $74.7 \%$; the area under the curve of MRI was $0.846(\mathrm{P}<0.05)$, the sensitivity was $84.6 \%$, and the specificity was $80.5 \%$; the area under the curve of CT combined with MRI was $0.947(\mathrm{P}<0.05)$, the sensitivity was $94.6 \%$, and the specificity was $86.5 \%$. In conclusion, the effect of dual-source CT perfusion imaging combined with MRI for examination of primary liver cancer is superior to that of single use of CT or MRI, and has high clinical application and popularization value.
\end{abstract}

Correspondence to: Dr Aixia Xu, Imaging Center, Affiliated Hospital of Weifang Medical University, 2428 Yuhe Road, Kuiwen, Weifang, Shandong 261031, P.R. China

E-mail: x5t6s8@163.com

Key words: dual-source CT perfusion imaging, primary liver cancer, MRI, hemodynamics

\section{Introduction}

Primary liver cancer is a relatively common malignant tumor. Intrahepatic cholangiocarcinoma and hepatocellular carcinoma are the most frequent types. They are characterized by insidious onset, rapid progression, low surgical resection rate, high degree of malignancy, and high recurrence rate $(1,2)$. Primary liver cancer has an excessively poor prognosis, the mortality rate of which ranks third among malignant tumors. If untreated, the survival time of patients is generally about three months. Once the symptoms of liver cancer appear, it often has already reached the advanced stage. Therefore, early diagnosis and timely treatment are key for prolonging the survival time of patients $(3,4)$. In recent years, imaging techniques have continuously developed. Imaging plays important roles in the diagnosis and treatment as well as follow-up of primary liver cancer. CT perfusion examination is a technique that is combined with traditional morphological imaging, which can determine the scope and size of the focus as well as changes in the surrounding organs. It can also measure tissue blood perfusion and capillary permeability (5). The changes of local and whole blood flow (BF) of the liver can be accurately observed by MRI perfusion imaging techniques, which can perform scans from multiple angles, with high sensitivity and no radiation damage (6). In this study, 63 patients with liver cancer were examined by CT and MRI, and the application values of CT perfusion imaging and MRI for the diagnosis of primary liver cancer were investigated. The details are below.

\section{Patients and methods}

Patients. Sixty-three patients with primary liver cancer who were admitted to the Affiliated Hospital of Weifang Medical University from February 2015 to May 2016 were randomly included. Inclusion criteria: i) patients who were diagnosed with primary liver cancer by surgery and pathology, ii) patients who had not undergone related antitumor therapies before CT and MRI examinations, including radiofrequency ablation, interventional embolization, chemotherapy, and radiotherapy, iii) patients who underwent CT and MRI examinations and iv) patients who signed the informed consent. Exclusion criteria: i) patients who had contraindications of CT and MRI scans, ii) patients who had intrahepatic and extrahepatic metastases and iii) patients who were complicated with mental, 
neurological, or other disorders. The general parameters of patients are shown in Table I. This study was approved by the Ethics Committee of the Affiliated Hospital of Weifang Medical University. Signed written informed consents were obtained from all participants before the study.

Preparation before examination. Patients were required to fast for $6 \mathrm{~h}$ before $\mathrm{CT}$ examination, and they were then informed of matters related to the examination. Breathing training (uniform, calm, shallow, and slow breathing) was provided for patients to avoid changes in the frequency of breathing resulting in poor image quality. The $18 \mathrm{G}$ intravenous detaining needle was embedded in advance, and patients were guided to drink $600 \mathrm{ml}$ of warm water at $30 \mathrm{~min}$ before scanning to fill the gastrointestinal tract.

CT examination. Patients were instructed to remove metallic foreign bodies, and maintain the supine position with hands raised on both sides of the headrest. A dual-source CT scanner (Siemens, München, Germany) was adopted to perform liver plain scan (tube voltage, $12 \mathrm{kV}$; tube current, $150 \mathrm{mAs}$; matrix, 512x512; alignment, $128 \times 0.6 \mathrm{~mm}$; and rotation time, $0.5 \mathrm{sec}$ ). After the scan was completed, the extent of foci and the plane that could display the largest lesions were determined. Perfusion examination was taken on the focus center layer which was selected as the targeted plane. An appropriate amount of water was provided for subjects to hydrate the injected contrast agent. Patients then took the same position as previously described, and were bound with compression by the abdominal belt of the instrument. Patients were told to maintain thoracic breathing, followed by injection with contrast agent using a high pressure automatic injector $[50 \mathrm{ml}(300 \mathrm{mg} / \mathrm{ml})$ iohexol (GE Healthcare, Dublin, Ireland; Approval No. Import Drug Registration Certificate No. H20090811)], with injection velocity of $5 \mathrm{ml} / \mathrm{sec}$. The perfusion scan was conducted at $6 \mathrm{sec}$ after injection. Before the beginning of the scan, patients were required to deeply inhale, followed by holding their breath for $30 \mathrm{sec}$, and the scan was taken once per second, for a total of 30 times. Body PCT model was selected for the equipment (tube current, $110 \mathrm{mAs}$; matrix, 512x512; alignment, $2 \times 32 \times 1.2 \mathrm{~mm}$; rotation time, $0.28 \mathrm{sec}$; and tube voltage, $80 \mathrm{kV}$ ). Image analysis and processing: the scanned images were transmitted to the processing workstation, and the perfusion plane that was clear and with minimal motion artifact was selected for analysis, followed by processing using the related software. The BF, blood volume (BV), permeability surface (PS), mean transit time (MTT), and hepatic arterial fraction (HAF) were calculated.

MRI examination. Patients were instructed to take supine position. First, an MR 3.0T HDX TwinSp Scanner (GE Healthcare, Little Chalfont, Buckinghamshire, UK) was used for routine scans. Scan extent: scanning of the whole liver, from the upper edge to the inferior edge of the liver. Routine sequence scan for the pelvic cavity was performed: i) conventional cross-section T1WI (TR, 106 msec; TE, 4.8 msec; matrix, 134x 256; FOV, $35 \times 35 \mathrm{~cm}$; layer distance, $2.4 \mathrm{~mm}$; slice thickness, $8 \mathrm{~mm}$; layer no. 20), imaging time, $28 \mathrm{sec}$; ii) sagittal plane T2WI (TR, 4,000 msec; TE, 100 msec; matrix, 230x256; FOV, 20×20 cm; layer distance, $0.6 \mathrm{~mm}$; slice thickness, $3.0 \mathrm{~mm}$; layer no. 19),
Table I. Basic parameters of patients.

\begin{tabular}{lc}
\hline Parameter & Subject $(\mathrm{n}=63)$ \\
\hline Mean age (years) & $61.78 \pm 6.54$ \\
Sex (male/female) & $38 / 25$ \\
Mean diameter of foci (cm) & $5.76 \pm 2.48$ \\
Multiple tumors (n, \%) & $17(26.98)$ \\
Single tumor (n, \%) & $46(73.02)$ \\
Education degree (n, \%) & \\
Junior middle school and below & $9(14.28)$ \\
Senior middle school and & $32(50.79)$ \\
technical secondary school & \\
Junior college and above & $22(34.92)$
\end{tabular}

imaging time, 3 min $42 \mathrm{sec}$; iii) high-resolution cross-section T2WI (TR, 6,620 msec; TE, 124 msec; matrix, 246x512; FOV, $20 \times 20 \mathrm{~cm}$; layer distance, $0.8 \mathrm{~mm}$; slice thickness, $4.0 \mathrm{~mm}$; layer no. 19), imaging time, $4 \mathrm{~min} 6 \mathrm{sec}$ and iv) coronal T2WI (TR, 6,410 msec; TE, 124 msec; matrix, 246x512; FOV, $20 \times 20 \mathrm{~cm}$; layer distance, $0.8 \mathrm{~mm}$; slice thickness, $4.0 \mathrm{~mm}$; layer no. 19), imaging time, 5 min $16 \mathrm{sec}$. Perfusion-weighted imaging (PWI) was conducted after the routine scan. By adopting a nuclear magnetic resonance double-tube high-pressure injector, $0.2 \mathrm{mmol} / \mathrm{kg}$ gadolinium-diethylenetriamine pentaacetic acid (Gd-DTPA; GE Healthcare; Approval No. Import Drug Registration Certificate No. H20100008) was injected with $2 \mathrm{ml} / \mathrm{sec}$ flow velocity in the first tube, while $20 \mathrm{ml}$ of normal saline was injected in the second tube. Fat-suppressed FLASH sequence was adopted for scanning coronal and cross sections: i) related parameters of PWI fatsuppressed FLASH (TR, 210 msec; TE, $1.2 \mathrm{msec}$; matrix, 256x208; FOV, 28x21 cm; layer distance, $4.0 \mathrm{~mm}$; slice thickness; $8.0 \mathrm{~mm}$; flip angle, $20^{\circ}$; layer no. 4 ; imaging time, $20 \mathrm{sec}$ and ii) technique parameters of perfusion imaging (TR, 107 msec; TE, 4.8 msec; matrix, 512x384; FOV, 36x $27 \mathrm{~cm}$; layer distance, $4.0 \mathrm{~mm}$; slice thickness, $8.0 \mathrm{~mm}$; layer no. 20 ; imaging time, $40 \mathrm{sec}$ ).

Evaluation indexes. Images were jointly read by two senior imaging physicians using a double-blinded method. Diagnostic accuracy rates were compared among different examination methods. Diagnostic accuracy rate $=$ detection number from diagnosis/final diagnosis number $\mathrm{x} 100 \%$. The parameters of dual-source CT perfusion included: i) BF volume passing through vascular structure and a certain amount of tissues per unit time; ii) BV in vascular structure and a certain amount of tissues; iii) PS, unidirectional transmission of contrast agent from capillary endothelium to intercellular space; iv) MTT, time of contrast agent flowing through vascular structure; and v) HAF of blood supply.

Processing of MRI data. The images were treated by processing software. The single layer with the largest number of tumor foci was selected, in which locations of enhancement areas containing mass, aorta, spleen, and necrotic tissue were measured, respectively, and portal venous perfusion (PVP), 

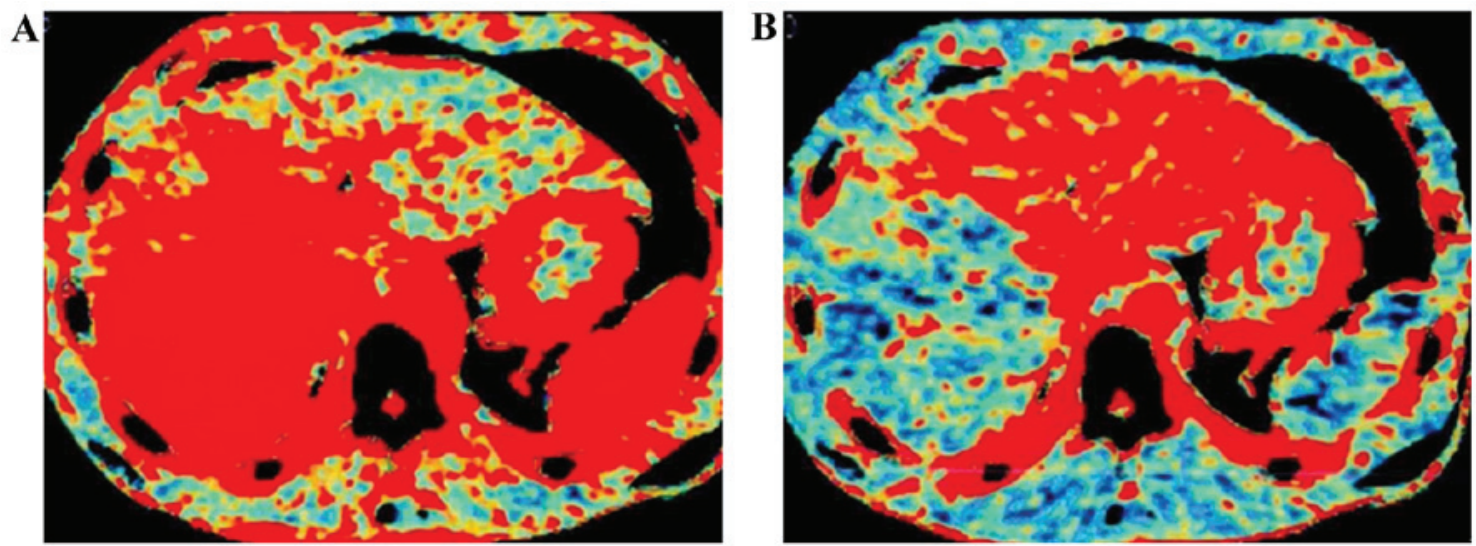

Figure 1. CT perfusion imaging. The patient is a 62-year-old male with primary liver cancer. (A) Hepatic artery perfusion image reveals that the arterial perfusion staining of the focus center is significantly higher than that of normal liver parenchyma in the distance; (B) venous perfusion image shows that the degree of venous perfusion staining of the focus center is lower than that of normal liver parenchyma, suggesting that the arterial perfusion volume of the focus center is higher than that of normal liver parenchyma, while venous blood supply shows the opposite.
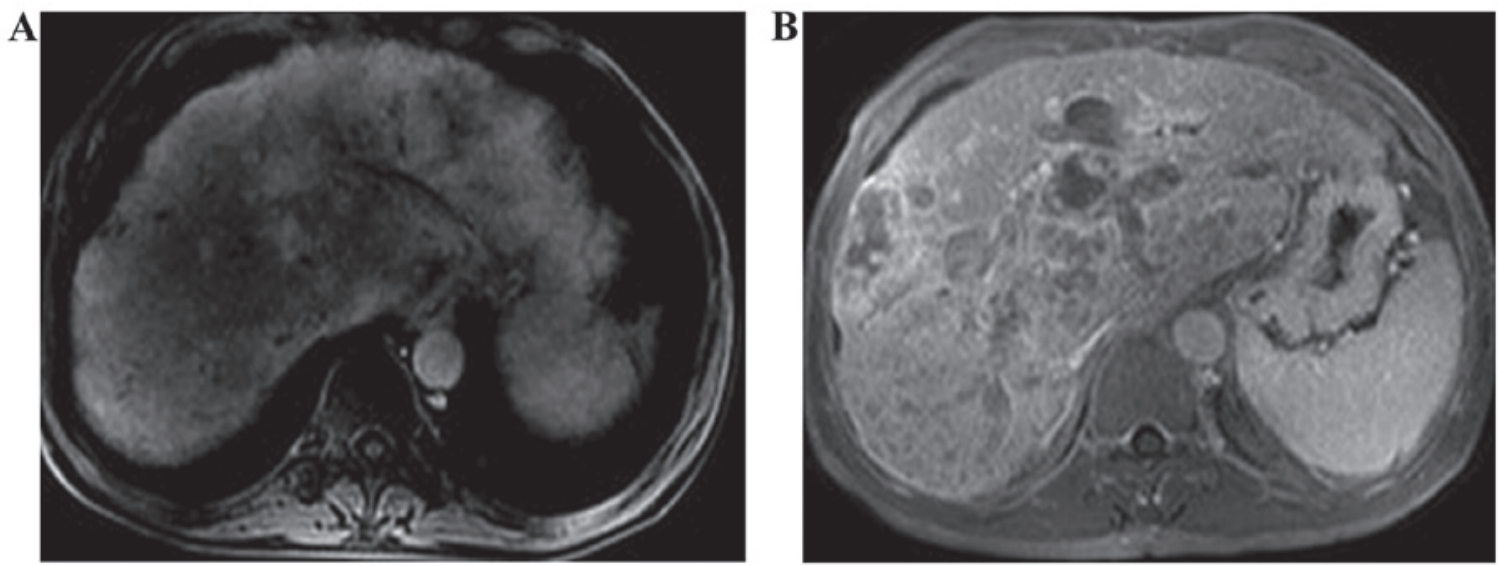

Figure 2. MRI perfusion imaging. The patient is a 62-year-old male with primary liver cancer. (A) PVP image of liver cancer of MRI perfusion reveals that the perfusion of the focus region is reduced and there is no clear boundary between the focus and surrounding liver parenchyma; (B) HAP image of liver cancer of MRI perfusion shows that non-uniform hyperperfusion was observable in the tumor.

hepatic perfusion index (HPI), hepatic arterial perfusion (HAP), and total liver perfusion (TLP) were obtained.

Statistical analysis. SPSS19.0 (SPSS, Inc., Chicago, IL, USA) software was used for data analysis. Numerical data are presented as mean \pm standard deviation and compared by t-test; CT, MRI, and combination of the two diagnostic methods were analyzed by ROC curves. $\mathrm{P}<0.05$ was considered statistically significant.

\section{Results}

Analysis of CT perfusion images. The CT perfusion images via level assignment revealed that the hyperchromatic degree of the focus center was significantly higher than that of normal liver parenchyma in the distance (Fig. 1); PVP images of liver cancer of MRI perfusion showed that the perfusion of the focus was reduced and there was no clear boundary between the focus and surrounding liver parenchyma (Fig. 2A); HAP of liver cancer of MRI perfusion showed that hyperperfusion was observable in tumors (Fig. 2B).
Analysis of parameters after dual-source CT perfusion scanning. There were no significant differences in PS and MTT between the focus center and normal liver parenchyma $(\mathrm{P}>0.05)$; BF, BV, and HAF of the focus center were significantly higher than those of normal liver parenchyma $(\mathrm{P}<0.05$; Table II).

Comparisons of MRI perfusion parameters in patients between the two groups. The results of MRI examination showed that HAP and HPI of the focus center were significantly higher than those of normal liver parenchyma; PVP of the focus center was significantly lower than that of normal liver parenchyma $(\mathrm{P}<0.05)$; the difference in TLP between the focus center and normal liver parenchyma was not significant $(\mathrm{P}>0.05$; Table III).

Comparisons of accuracy rates of different detection methods for primary liver cancer. The accuracy rates of dual-source CT perfusion imaging, MRI examination, and combined examination of CT and MRI for primary liver cancer were $76.19 \%$ (48/63), 85.71\% (54/63) and 95.24\% (60/63), 
Table II. Comparisons of CT perfusion parameters in patients between the two groups.

\begin{tabular}{lcccccc}
\hline Group & Case & BF $(\mathrm{ml} / 100 \mathrm{ml} / \mathrm{min})$ & $\mathrm{BV}(\mathrm{ml} / \mathrm{l})$ & $\mathrm{MTT}(\mathrm{sec})$ & $\mathrm{HAF}$ & $\mathrm{PS}(0.5 \mathrm{ml} / 100 \mathrm{ml} / \mathrm{min})$ \\
\hline Focus center & 63 & $121.65 \pm 21.43$ & $175.68 \pm 33.26$ & $143.29 \pm 13.27$ & $0.35 \pm 0.07$ & $112.62 \pm 14.53$ \\
Normal liver parenchyma & 63 & $79.48 \pm 9.37$ & $113.45 \pm 13.63$ & $145.15 \pm 12.34$ & $0.13 \pm 0.04$ & $113.49 \pm 14.47$ \\
t-test & & 14.311 & 13.742 & 0.815 & 24.905 & 0.337 \\
P-value & & $<0.0001$ & $<0.0001$ & 0.4168 & $<0.0001$ & 0.7369 \\
\hline
\end{tabular}

BF, blood flow; BV, blood volume; MTT, mean transit time; HAF, hepatic arterial fraction; PS, permeability surface.

Table III. Comparisons of MRI perfusion parameters in patients between the two groups.

\begin{tabular}{|c|c|c|c|c|c|}
\hline Group & Case & $\operatorname{PVP}(\mathrm{ml} / 100 \mathrm{ml} / \mathrm{min})$ & $\mathrm{HAP}(\mathrm{ml} / 100 \mathrm{ml} / \mathrm{min})$ & HPI (\%) & $\mathrm{TLP}(\mathrm{ml} / 100 \mathrm{ml} / \mathrm{min})$ \\
\hline Focus center & 63 & $8.63 \pm 2.42$ & $45.64 \pm 7.25$ & $84.26 \pm 3.23$ & $58.52 \pm 8.43$ \\
\hline Normal liver parenchyma & 63 & $23.43 \pm 8.34$ & $33.47 \pm 6.62$ & $58.18 \pm 2.37$ & $60.69 \pm 8.48$ \\
\hline t-test & & 13.527 & 9.839 & 51.671 & 1.440 \\
\hline P-value & & $<0.0001$ & $<0.0001$ & $<0.0001$ & 0.1523 \\
\hline
\end{tabular}

PVP, portal venous perfusion; HAP, hepatic arterial perfusion; HPI, hepatic perfusion index; TLP, total liver perfusion.

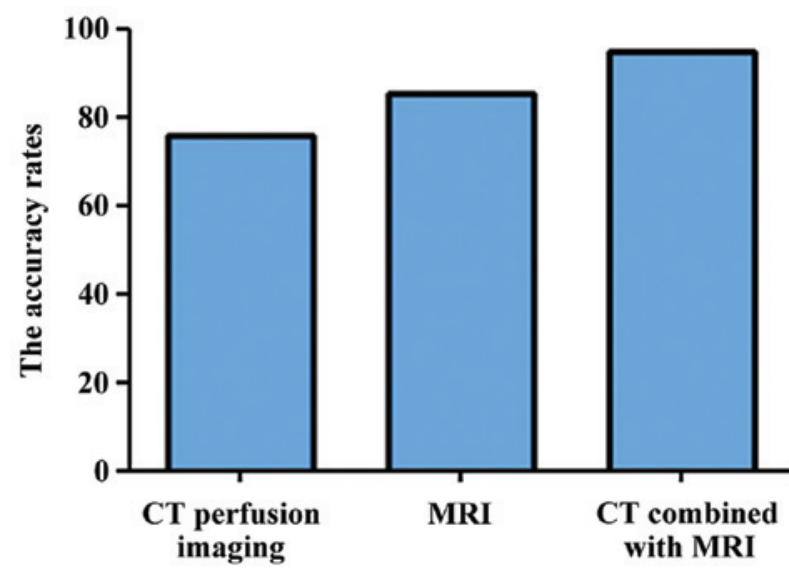

Figure 3. Accuracy rates of different detection methods; the differences between the three methods were statistically significant, $\mathrm{P}<0.05$.

respectively; the differences were statistically significant $(\mathrm{P}<0.05$; Fig. 3).

Diagnosis of primary liver cancer. The area under the curve of CT perfusion imaging examination was $0.753(\mathrm{P}<0.05)$, the sensitivity was $79.2 \%$, and the specificity was $74.7 \%$; the area under the curve of MRI was $0.846(\mathrm{P}<0.05)$, the sensitivity was $84.6 \%$, and the specificity was $80.5 \%$; the area under the curve of CT combined with MRI was $0.947(\mathrm{P}<0.05)$, the sensitivity was $94.6 \%$, and the specificity was $86.5 \%$ (Fig. 4).

\section{Discussion}

Primary liver cancer is a tumor with enrichment of blood vessels, which generally manifests as liver pain, anorexia, ascites, fatigue, weight loss, jaundice, and other clinical symptoms. In severe cases, coma and systemic failure can occur (7). The liver is a unique organ whose blood can be doubly supplied by the portal vein and hepatic artery. The portal vein is the main blood supply pathway in the normal state, while blood supply from the hepatic artery plays an important role in angiogenesis during the occurrence and progression of primary liver cancer (8). A large number of proangiogenic growth factors can be secreted by liver tumor tissue, which leads to intrastromal blood sinus formation with gradual vascularization, and shows uncontrolled growth, thereby causing the gradual increase of hepatic artery blood supply and the gradual decrease of portal blood supply, ultimately resulting in high arterial blood supply (9). The diagnosis of primary liver cancer is usually performed by direct and rapid imaging examinations in clinical practice, including color Doppler ultrasonography, CT, and MRI (10). Ultrasonography has the advantages of no trauma, no pain, quick and simple inspection, and repeatability. However, it also has disadvantages, such as low resolution, and sensitivity to the effects of the heartbeat or intestinal gas (the left half of the liver is greatly affected). In addition, ultrasonography is not sensitive to lesions with low blood-flow perfusion or lack of blood supply. Therefore, examinations using CT and MRI for diagnosis are mainly adopted in clinical practice (11).

Perfusion refers to oxygen and nutrients being transported to tissues and cells by BF through capillary networks, and can reflect the hemodynamics and functions of organs and tissues (12). The earliest investigations involving nuclear medicine were aimed at local tissue perfusion imaging. CT perfusion imaging was first proposed in the 1990s. It involves intravenous bolus injection of a contrast agent, and single-level dynamic scanning of the selected layers to obtain time-density curves (TDC) that reflect BF characteristics; BF, BV, MTT, PS. Other perfusion parameters are calculated 
A

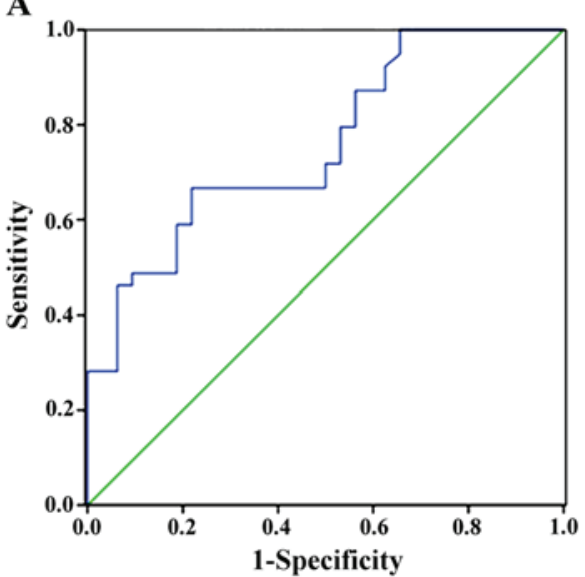

B

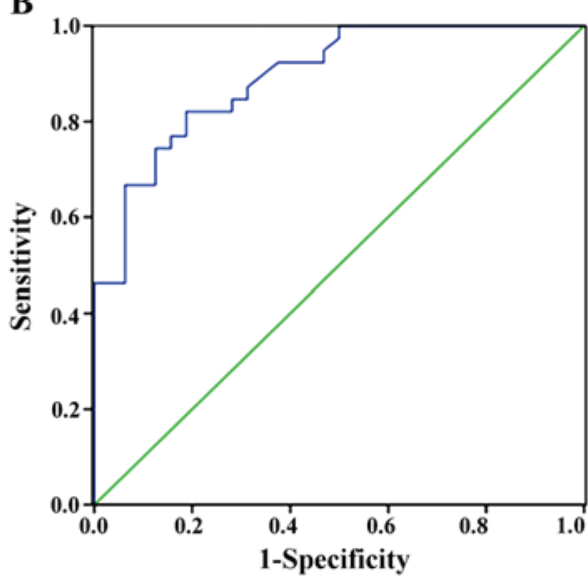

C

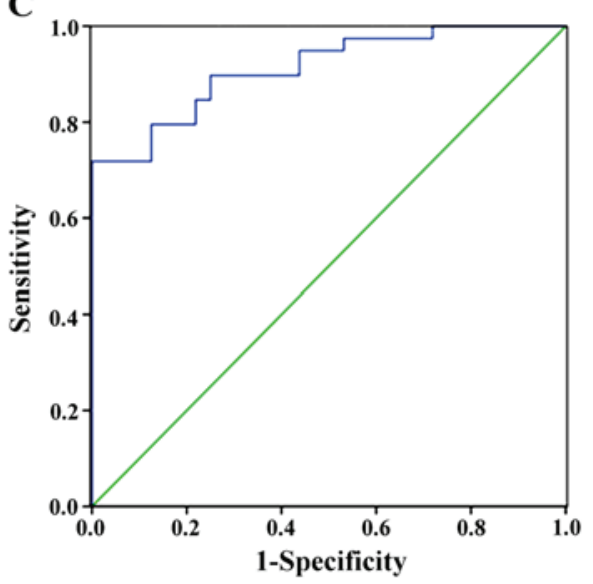

Figure 4. (A) ROC curve of CT perfusion imaging examination; for CT examination, the area under the ROC curve is 0.753 . (B) ROC curve of MRI examination; for MRI examination, the area under the ROC curve is 0.846. (C) ROC curve of combined examination of CT and MRI; for CT combined with MRI, the area under the ROC curve is 0.947 . Comparing the three methods, the combined examination of CT and MRI is significantly more sensitive than the other two methods.

by related professional software according to TDC, followed by level assignment to form perfusion images, which can observe the border and essence of the focus, and directly and comprehensively determine whether there is necrosis and other aspects of the focus. In addition, it can determine vascular characteristics and BF perfusion characteristics (13). Perfusion imaging techniques have been continuously developed in recent years. Scanning speed, imaging coverage area, and scanning methods have changed greatly (14). By adopting a second-generation dual-source CT scanner (Siemens) to conduct perfusion imaging and scans in this study, we showed that the hyperchromatic degree of the focus center was significantly higher than that of normal liver parenchyma in the distance. Through analysis of perfusion parameters, the $\mathrm{BF}$ and $\mathrm{BV}$ values were $121.65 \pm 21.43 \mathrm{ml} / 100 \mathrm{ml} / \mathrm{min}$ and $175.68 \pm 33.26 \mathrm{ml} / 1$, respectively in the focus center, and $79.48 \pm 9.37 \mathrm{ml} / 100 \mathrm{ml} / \mathrm{min}$ and $113.45 \pm 13.63 \mathrm{ml} / 1$, respectively, in normal liver parenchyma. The values of the two parameters in the focus center were significantly higher than those in normal liver parenchyma, which was because of incomplete basement membranes of new vessels with irregular morphology, high vascular permeability, small vascular resistance, and large BF. Therefore, when contrast agent passes through new tumor vessels, the amount and flow rate of contrast agent per unit volume will elevate simultaneously, thereby increasing BF and BV (15). The MTT and PS values in the focus center were $143.29 \pm 13.27 \mathrm{sec}$ and $112.62 \pm 14.53,0.5 \mathrm{ml} / 100 \mathrm{ml} / \mathrm{min}$, respectively. The MTT and PS values of the normal liver parenchyma were $145.15 \pm 12.34 \mathrm{sec}$ and $113.49 \pm 14.47,0.5 \mathrm{ml} / 100 \mathrm{ml} / \mathrm{min}$, respectively. The differences with these two parameters were not significant $(\mathrm{P}>0.05)$. During CT perfusion examination, because of the often inadequate cooperation of breathing in patients, the drift phenomenon of images occurs easily, sometimes resulting in the images being unable to be processed. Although the demand for radiation dose is decreasing with the continuous improvement of CT techniques, it remains the biggest drawback, which may cause increased risk of tumors induced by radiation in patients (16).
MRI has been applied for the diagnosis of liver cancer since the 1980, and has the advantages of multi-parameter imaging and high resolution of soft tissue, with no iodine allergy and radiation hazards (17). Lesions can primarily be observed by MRI plain scan, which provides related information for MRI perfusion; the distribution and change of microcirculation in tissue as well as the difference in BF perfusion between local abnormal tissue and normal tissue can be determined by MRI perfusion examination. Therefore, the activity and function of local lesions can be evaluated (18). After intravenous bolus injection of the paramagnetic contrast agent, Gd-DTPA, gradient echo signals acquired by reverse switching of the coil is read through PWI fat-suppressed FLASH sequences followed by excitation via a small angle. The perfusion map shows that high perfusion can be observed when contrast agent passes through the capillaries of tissues, which are short T1 short T2 signals; moreover, the T2 value is shortened more significantly (19). The results of this study revealed that there was no significant difference in TLP between the focus center and normal liver parenchyma $(\mathrm{P}>0.05)$. HAP and HPI of the focus center were significantly higher than those of normal liver parenchyma, but PVP of the focus center was significantly lower than that of normal liver parenchyma $(\mathrm{P}<0.05)$, which was because the blood of foci in primary liver cancer is mainly supplied by the hepatic artery with especially fast flow velocity, thereby increasing the pressure. It is therefore difficult for the $\mathrm{BF}$ of the portal vein to enter the focus center. Blood supplied by the portal vein is minimal, which is the opposite to that seen in normal liver tissue. Therefore, perfusion imaging shows relatively little perfusion in the portal vein of the focus center, while there is more perfusion in the hepatic artery. The HAP index is therefore higher. There are few factors that can interfere with MRI examination, while its image quality is only affected by breathing. Inadequate cooperation of breathing in patients easily causes failure of examination (20).

At present, the clinical diagnosis of primary liver cancer is often affected by many factors, such as scanning technique and the patients themselves, which causes atypical imaging performance, thereby limiting diagnosis. Therefore, the combined 
examination of CT and MRI is generally selected to improve the diagnostic rate of primary liver cancer in clinical practice. In this study, there were 60 patients confirmedly diagnosed with primary liver cancer by the combined examination of CT and MRI, with accuracy rate of $95.24 \%$. The area under the curve of CT combined with MRI was 0.947, which was significantly higher than 0.753 of CT perfusion imaging and 0.846 of MRI examination $(\mathrm{P}<0.05)$. The combined examination of $\mathrm{CT}$ and MRI, which is conducive to the early diagnosis and clinical staging diagnosis of primary liver cancer, is a sensitive diagnostic method that can promote the use of perfusion imaging in clinical practice, and no longer only in scientific research.

In conclusion, the combined examination of CT and MRI is superior to single CT perfusion imaging or MRI examination for the diagnosis of primary liver cancer. It has a high clinical application value and is worthy of wider use and application.

\section{References}

1. Younossi ZM, Otgonsuren M, Henry L, Venkatesan C, Mishra A, Erario $M$ and Hunt $S$ : Association of nonalcoholic fatty liver disease (NAFLD) with hepatocellular carcinoma (HCC) in the United States from 2004 to 2009. Hepatology 62: 1723-1730, 2015.

2. Lee JM, Park JW and Choi BI: 2014 KLCSG-NCC Korea Practice Guidelines for the management of hepatocellular carcinoma: HCC diagnostic algorithm. Dig Dis 32: 764-777, 2014

3. Jemal A, Bray F, Center MM, Ferlay J, Ward E and Forman D: Global cancer statistics. CA Cancer J Clin 61: 69-90, 2011.

4. Hann HW, Coben R, Brown D, Needleman L, Rosato E, Min A, Hann RS, Park KB, Dunn S and DiMarino AJ: A long-term study of the effects of antiviral therapy on survival of patients with HBV-associated hepatocellular carcinoma (HCC) following local tumor ablation. Cancer Med 3: 390-396, 2014

5. Kanda T, Yoshikawa T, Ohno Y, Fujisawa Y, Kanata N, Yamaguchi M, Seo Y, Yano Y, Koyama H, Kitajima K, et al: Perfusion measurement of the whole upper abdomen of patients with and without liver diseases: Initial experience with 320-detector row CT. Eur J Radiol 81: 2470-2475, 2012.

6. Vardal J, Salo RA, Larsson C, Dale AM, Holland D, Groote IR and Bjørnerud A: Correction of B0-distortions in echo-planar-imaging-based perfusion-weighted MRI. J Magn Reson Imaging 39: 722-728, 2014.

7. Wang W, Cheng J, Qin JJ, Voruganti S, Nag S, Fan J, Gao Q and Zhang R: RYBP expression is associated with better survival of patients with hepatocellular carcinoma (HCC) and responsiveness to chemotherapy of HCC cells in vitro and in vivo. Oncotarget 5: 11604-11619, 2014.
8. Kim KW, Lee JM, Kim JH, Klotz E, Kim HC, Han JK and Choi BI: CT color mapping of the arterial enhancement fraction of VX2 carcinoma implanted in rabbit liver: comparison with perfusion CT. AJR Am J Roentgenol 196: 102-108, 2011.

9. Watanabe K, Yamamoto T, Sugimoto $\mathrm{H}$ and Yamamoto T: A study on blood flow characteristics of hepatic vein. In: 6th World Congress on Biomechanics (WCB 2010), 1-6 August 2010, Singapore. Goh Cho Hong J and Lim CT (eds). Vol 31. 1st edition. Springer Berlin-Heidelberg, Berlin, pp1475-1478, 2010.

10. Hong YM, Yoon KT, Cho M, Heo J, Woo HY and Lim W: A case of small hepatocellular carcinoma with an extensive lymph node metastasis at diagnosis. Clin Mol Hepatol 20: 310-312, 2014.

11. Zha Y, Zhou M, Hari A, Jacobsen B, Mitragotri N, Rivas B, Ventura OG, Boughton J and Fox JC: Ultrasound diagnosis of malaria: examination of the spleen, liver, and optic nerve sheath diameter. World J Emerg Med 6: 10-15, 2015.

12. George RT, Mehra VC, Chen MY, Kitagawa K, Arbab-Zadeh A, Miller JM, Matheson MB, Vavere AL, Kofoed KF, Rochitte CE, et al: Myocardial CT perfusion imaging and SPECT for the diagnosis of coronary artery disease: a head-to-head comparison from the CORE320 multicenter diagnostic performance study. Radiology 272: 407-416, 2014.

13. Kanda T, Yoshikawa T, Ohno Y,Kanata N, Koyama H, Takenaka D and Sugimura K: CT hepatic perfusion measurement: comparison of three analytic methods. Eur J Radiol 81: 2075-2079, 2012.

14. Wang R, Yu S, Alger JR, Zuo Z, Chen J, Wang R, An J, Wang B, Zhao J, Xue R, et al: Multi-delay arterial spin labeling perfusion MRI in moyamoya disease - comparison with CT perfusion imaging. Eur Radiol 24: 1135-1144, 2014.

15. Wang Q, Shi G, Wang L, Liu X and Wu R: Early prediction of response of sorafenib on hepatocellular carcinoma by CT perfusion imaging: an animal study. Br J Radiol 87: 20130695-20130695, 2014.

16. Sahinarslan A, Erbas G, Kocaman SA, Bas D, Akyel A, Karaer D, Ergun MA, Arac M and Boyaci B: Comparison of radiation-induced damage between $\mathrm{CT}$ angiography and conventional coronary angiography. Acta Cardiol 68: 291-297, 2013.

17. Ganten MK, Schuessler M, Bäuerle T, Muenter M, Schlemmer HP Jensen A, Brand K, Dueck M, Dinkel J, Kopp-Schneider A, et al: The role of perfusion effects in monitoring of chemoradiotherapy of rectal carcinoma using diffusion-weighted imaging. Cancer Imaging 13: 548-556, 2013.

18. Simonsen CZ, Madsen MH, Schmitz ML, Mikkelsen IK, Fisher $M$ and Andersen G: Sensitivity of diffusion- and perfusion-weighted imaging for diagnosing acute ischemic stroke is $97.5 \%$. Stroke 46: 98-101, 2015.

19. Asayama Y, Tajima T, Nishie A, Ishigami K, Kakihara D, Nakayama T, Okamoto D, Fujita N, Aishima S, Shirabe K, et al: Uptake of Gd-EOB-DTPA by hepatocellular carcinoma: radiologic-pathologic correlation with special reference to bile production. Eur J Radiol 80: e243-e248, 2011.

20. Garrett R: Solid liver masses: approach to management from the standpoint of a radiologist. Curr Gastroenterol Rep 15: 359-359, 2013. 